\title{
Long-term retention as a function of word concreteness under conditions of free recall*
}

\author{
LEO POSTMAN and SHEILA BURNS \\ University of California, Berkeley, California 94720
}

\begin{abstract}
Acquisition and long-term retention of concrete (C) and abstract (A) words were investigated under conditions of multiple-trial free recall. Both unmixed and mixed lists were used in original learning. Retention was tested either $1 \mathrm{~min}$ or 1 week after attainment of the learning criterion. Acquisition was faster and retention was higher for $\mathrm{C}$ than for $\mathrm{A}$ words. These differences were more pronounced for mixed than for unmixed lists.
\end{abstract}

Task variables, such as meaningfulness and intralist similarity, which influence speed of acquisition usually do not have corresponding effects on long-term retention. Rather, the level of retention is likely to be invariant when degree of learning has been equated (cf. Postman, 1971). A deviation from this typical picture was observed in a recent study (Postman \& Burns, 1973) of the long-term retention of paired-associate lists as a function of the concreteness-imagery (C-I) value of the stimulus and the response terms. As usual, increases in C-I value facilitated acquisition, and more so on the stimulus than on the response side (cf. Paivio, 1971, Chap. 6). With degree of learning equated by adjustment in the number of training trials, there was a drastic change in the rank order of the conditions on a test of retention after 1 week. Recall was lowest when the C-I value of both the stimuli and the responses was high. This reversal implies that reliance on imaginal mediators maximizes difficulties of decoding at the time of retrieval. There were indications, however, that recall of the responses per se may be positively related to C-I value. If such a relationship does, indeed, exist, comparable differences may be expected on a test of retention for lists of words acquired under conditions of free recall.

Free recall learning is more rapid for concrete than for abstract words (Paivio, 1971, Chap. 7). If the advantage of concrete words derives from a higher probability of imaginal encoding, there must be sources of facilitation that outweigh the decoding difficulties attendant upon retrieval from imaginal transformations. The same applies to the superior recall of pictures as compared to their noun labels. A number of different mechanisms have been proposed to account for the enhancement of free recall by imaginal encoding (cf. Paivio \& Csapo, 1973). One hypothesis, for which there is some empirical support, is that imaginal representations can be unitized more easily than strictly verbal ones, so that the

\footnotetext{
*This research was supported by Grant MH-12006 from the
} National Institute of Mental Health. retrieval of higher-order units is facilitated (Begg, 1972). However, the degree of subjective organization as indexed by measures of output consistency is not dependably higher for concrete than for abstract words (Paivio, Yuille, \& Rogers, 1969). An alternative hypothesis is that the possibility of successful retrieval is increased by the dual encoding-imaginal as well as verbal-which becomes increasingly likely as one moves from abstract to concrete words and then to pictures. There is some presumptive evidence, albeit only indirect, for this interpretation (Paivio \& Csapo, 1973). Finally, it is conceivable that imaginal representations constitute stronger or more accessible traces than verbal ones. The differences between picture and word recall observed by Paivio and Csapo are consistent with this conclusion. While this last hypothesis merely restates the explanatory problem, it has to be considered seriously if the empirical facts continue to point in this direction.

None of these hypotheses necessarily implies that long-term retention should be higher for lists of concrete than of abstract words when degree of learning is equated (cf. Paivio, in press). It is possible that the subjective organization of concrete words, presumably based at least in part on imaginal representations, is more persistent than the organization of abstract words. This possibility can be evaluated directly, within the limitations of available indices of organization. If dual encoding is the effective sóurce of facilitation in acquisition, the consequences for retention will depend in the first instance on whether the two kinds of memorial representations are lost or become degraded concomitantly. If they are, higher retention of concrete words is not to be expected; if the losses evolve independently, dual encoding should increase the probability of delayed retrieval mediated by at least one of the representations. The assumption of independent loss is not very plausible in view of the strong associative connection between the two types of encoding. Finally, imaginal representations may be inherently more durable or more resistant to interference than verbal ones. The latter hypothesis and the principle of dual encoding are not mutually 
exclusive, and either is compatible with differences in the degree and persistence of subjective organization. It will obviously be difficult to decide among these alternatives which may operate singly or in combination.

Before the theoretical possibilities can be narrowed down, it is necessary to determine whether there are, in fact, differences in long-term retention between $\mathrm{C}$ and $\mathrm{A}$ words learned under conditions of free recall. There is as yet no acceptable evidence bearing on this question. The results of a recent experiment by Begg and Robertson (1973) can be discounted on methodological grounds, and in particular because of the failure to equate appropriately degree of original learning. (Their finding of a concreteness effect in paired-associate retention is likewise vitiated by the use of a biased technique for controlling the terminal level of acquisition.) The objections to the procedures used by Begg and Robertson, which preclude an evaluation of retention differences, have been detailed elsewhere (Postman, in press). Thus, the question of whether free recall declines more rapidly for $\mathrm{A}$ than for $\mathrm{C}$ lists has remained open.

Both unmixed and mixed lists were used in the present experiment. The majority of previous studies comparing immediate recall of concrete and of abstract words have used mixed lists. Differences between distinct classes of items in a mixed list may reflect, to an unknown degree, Ss' learning preferences (Underwood, 1964, p. 68). Paivio, Yuille, and Rogers (1969) used unmixed as well as mixed lists and found higher recall for concrete than for abstract words under both conditions. However, the unmixed lists were only half as long as the mixed ones, so that the magnitude of the differences cannot be compared directly. Thus, a further evaluation of the results obtained under the two conditions of learning was considered useful. It was also of interest to determine whether the acquisition procedure would have a systematic effect on long-term retention. In particular, differences in retention related to the degree of organization should be less pronounced for mixed than for unmixed lists. Such differences should be attenuated for mixed lists, since groupings comprising members of both classes are likely to be formed during acquisition.

\section{METHOD}

\section{Design}

The experimental design comprised eight groups which represented the combination of four conditions of learning with two retention intervals $(1 \mathrm{~min}$ and 1 week). Unmixed lists of concrete or abstract words were used in two of the conditions. Mixed lists, in which half the items were concrete and half were abstract, were used in the other two conditions. While exactly the same materials were presented in the two mixed-list conditions, the criterion of acquisition was defined with respect to the subset of concrete words in one case and with respect to the subset of abstract words in the other. These subsets were also present in the corresponding unmixed lists. Thus, it was possible to compare performance on the same items learned to a common criterion in the context of unmixed and of mixed lists. Since differences in the rate of acquisition were expected, the 1-min test provided a baseline for the assessment of long-term retention. The unmixed-list conditions will be designated as $\mathrm{C}$ and $A$ and the mixed-list conditions as $C / A$ and $A / C$, with the first letter referring to the critical and the second letter to the noncritical set of items. The letters I (immediate) and D (delayed) will be used to refer to the 1-min and 1-week retention tests, respectively.

\section{Lists}

The learning materials were 64 concrete words and 64 abstract words selected from the norms of Paivio, Yuille, and Madigan (1968). Each set of 64 words was subdivided in to four subsets of 16 items each. For the subsets of concrete words, the mean C values ranged from 6.18 to 6.45 , and the mean I values from 5.48 to 5.85 . The corresponding ranges for the subsets of abstract words were 2.24-2.80 and 3.07-3.42. All eight subsets were equated as closely as possible with respect to the following attributes: $m$ value, word frequency, number of syllables per word, number of different first letters, and number of letters per word.

All lists consisted of 32 words. There were four different unmixed lists of a given type ( $C$ or $A$ ), with each subset included in two of the lists. There were eight different mixed lists, with each of the eight subsets (four $\mathrm{C}$ and four $\mathrm{A}$ ) again included in two of the lists. All lists were used an equal number of times. Within each of the unmixed and the mixed lists, one subset was designated as critical. Each subset was used as the critical one equally often.

\section{Procedure}

Study and test trials were alternated during original learning. On study trials, the items were presented at a 3-sec rate. There were six different orders of presentation. A number of restrictions were observed in the assignment of words to positions: (a) eight items from each subset occurred in each half of the list; (b) no more than two items from the same subset occurred in sequence; (c) the first and the last position were occupied by items from different subsets; (d) across orders, the first and last positions were occupied by items from each subset half the time; (e) no item appeared in the same position more than once; (f) no forward or backward sequences were repeated.

On test trials, Ss were given $3 \mathrm{~min}$ in which to write down all the words they could remember in any order they wished. There were 32 lines on each test sheet. The Ss called out the words they were writing down, so that $E$ could keep track of the number of items recalled. Practice on the unmixed list was continued to a criterion of $28 / 32$, with the additional requirement that $14 / 16$ of the items in the critical subset be recalled on the terminal trial. In the mixed-list conditions, the criterion was $14 / 16$ on the critical subset. The dual criterion was used for the unmixed lists in order to ensure that (a) practice on the $C$ and $A$ lists would be carried to the same level and (b) recall could be tested for the same critical items as in the mixed lists. Under this arrangement, criterion on the critical subset of an unmixed list could be reached before the terminal trial on which the dual requirement was satisfied. Consequently, degree of learning for the critical subset might be somewhat higher in the unmixed than in the mixed lists. This fact would, of course, not bias comparisons between Lists $\mathrm{C}$ and $\mathrm{A}$ or between Lists C/A and $A / C$. As far as differences in retention between corresponding subsets in unmixed and mixed lists are concerned, the immediate test of recall would permit an assessment of possible discrepancies in the terminal level of learning.

A retention test was administered either $1 \mathrm{~min}$ or 1 week af ter attainment of the criterion. There were three successive test trials of $3 \mathrm{~min}$ each. The recall procedure was exactly the same as in original learning. The Ss were not given any feedback between test trials. If $S$ had failed to write down 32 words on the third trial, he was urged to fill each of the empty spaces, even if he had to guess in order to do so. This terminal phase of the test was unpaced. 
Subjects

There were $16 \mathrm{Ss}$ in each of the eight groups. The Ss were students at the University of California who were not necessarily naive to verbal learning experiments. A randomized block procedure was used in the assignment of $S$ s to conditions.

\section{RESULTS}

The measures of acquisition and retention will first be analyzed separately for unmixed and mixed lists, and the difference between the two types of list will be evaluated thereafter. All analyses will be based on independent groups. Thus, the scores on the critical subsets of Lists $\mathrm{C} / \mathrm{A}$ and $\mathrm{A} / \mathrm{C}$ will be used in comparisons of concrete and abstract words learned under mixed-list conditions. Scores on the critical subsets will also be used in evaluations of the differences between unmixed and mixed lists.

\section{Acquisition}

Performance on the first test trial of original learning is of interest because preferences under the mixed-list procedure are likely to become apparent early in acquisition. The mean scores are presented in Table 1. The difference between the two classes of items is small and not significant both for total unmixed lists and for the critical subsets therein $(\mathrm{Fs}<1)$. The relative difference is substantially larger in the case of the critical subsets of the mixed lists and is significant at the .01 level $[F(1,62)=8.40]$. When the corresponding subsets in both types of list are considered, the Type of List by Item Class interaction falls, however, short of significance $[F(1,124)=2.05, p>.05]$.

Essentially the same picture emerges when the trials on which individual words were recalled for the first time are examined. The mean trial of first recall was calculated for each $\mathrm{S}$, and the means of the means were as follows: unmixed $\mathrm{C}-2.25$ (critical subset-2.31); unmixed A-2.40 (critical subset-2.49); mixed C-2.29; mixed A-2.77. In the unmixed-list condition, there is only a small separation between $\mathrm{C}$ and $\mathrm{I}$ items; the $\mathrm{F}$ ratios are 1.39 and 1.08 for total lists and critical subsets, respectively. When the critical subsets of the mixed lists are considered, however, the difference is clearly significant $[F(1,62)=9.28, p<.01]$. The Type of List by Item Class interaction is not significant $(F=$ 1.74).

The mean numbers of trials to criterion are

Table 1

Mean Number Correct on First Test Trial of Learning

\begin{tabular}{ccccc} 
& \multicolumn{2}{c}{ Unmixed Lists } & & Mixed Lists \\
\cline { 2 - 3 } Item & & Critical & & $\begin{array}{c}\text { Critical } \\
\text { Class }\end{array}$ \\
\hline C & Total & Subset & & Subset \\
A & 11.03 & 5.63 & 6.16 \\
\hline
\end{tabular}

Note-MS $S_{E}$ are 11.88 for total unmixed lists, 5.06 for critical subsets of unmixed lists, and 4.84 for critical subsets of mixed lists.
Table 2

Mean Trials to Criterion

\begin{tabular}{|c|c|c|c|c|c|c|}
\hline \multirow{3}{*}{$\begin{array}{l}\text { Item } \\
\text { Class }\end{array}$} & \multicolumn{4}{|c|}{ Unmixed Lists } & \multirow{2}{*}{\multicolumn{2}{|c|}{$\frac{\text { Mixed Lists }}{\text { Critical Subse }}$}} \\
\hline & \multicolumn{2}{|c|}{ Total } & \multicolumn{2}{|c|}{ Critical Subset } & & \\
\hline & I & $\mathrm{D}$ & I & $\mathrm{D}$ & I & D \\
\hline $\mathrm{C}$ & 5.94 & 5.50 & 5.06 & 5.06 & 4.56 & 6.06 \\
\hline A & 7.50 & 7.38 & 6.06 & 6.44 & 8.25 & 6.81 \\
\hline
\end{tabular}

Note-MS $S_{E}$ are 9.24 for total unmixed lists, 7.25 for critical subsets of unmixed lists, and 9.61 for critical subsets of mixed lists.

summarized in Table 2. For unmixed lists, there were two criteria, namely, 28/32 on the entire list and 14/16 on the critical subset; for mixed lists, there was only the latter. At each criterion, acquisition was faster for $\mathrm{C}$ than for A items. In the analyses of these results, retention irterval (I or D) was included as a "dummy" variable; but item class was the only significant source of variance. For the criterion of $28 / 32$ on unmixed lists, the value of $F(1,60)$ is $5.11(\mathrm{p}<.05)$; when the criterion of $14 / 16$ on the critical subsets is considered, the corresponding $F$ ratios are $3.11(p>.05)$ for the unmixed lists and $8.20(\mathrm{p}<.01)$ for the mixed lists. The $\mathrm{C}$-I value of the words is again found to have a more pronounced effect on mixed-list than on unmixed-list learning. Once more, however, the interaction is not significant $(F=1.01)$.

The scores on the final learning trial were comparable under the various conditions. The mean numbers correct were as follows: C-I- 28.81 (critical subset-14.69); C-D-28.88 (critical subset-14.94); A-I-29.56 (critical subset-14.94); A-D-28.75 (critical subset-14.63); C/A-I-14.56; C/A-D-14.62; A/C-I-14.43; A/C-D_-14.25.

\section{Organization During Acquisition}

A measure of intertrial repetition (ITR) was used to assess the degree of subjective organization developed during acquisition. Pairwise repetitions (for all items recalled, regardless of subset) were determined between the trial preceding attainment of the criterion and the criterial trial. The difference between the observed number of repetitions and that expected by chance (Bousfield \& Bousfield, 1966) was divided by the maximum possible number minus the expected value, yielding a relative measure of output consistency. The mean ITR scores are presented in Table 3 . The overall mean is higher for the mixed than for the unmixed lists $[F(1,124)=7.53, p<.01]$. There are, however, no significant differences between either Lists $\mathrm{C}$ and $\mathrm{A}$ or between Lists $\mathrm{C} / \mathrm{A}$ and $\mathrm{A} / \mathrm{C}(\mathrm{Fs}<1)$. Thus, there is no evidence that the terminal degree of organization is related to $\mathrm{C}$-I value.

For the mixed lists, an analysis of runs (Frankel \& Cole, 1971) was carried out to measure the extent of clustering by class of items on the criterial trial. A small number of Ss (three in Condition C/A-I and one in 


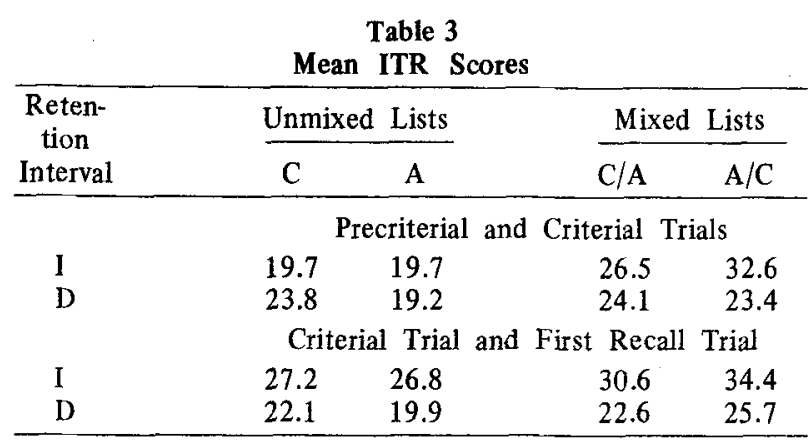

Note-MS $S_{E}$ are 15.64 for measures in top half of the table and 15.76 for measures in bottom half.

Condition A/C-D) who reproduced the items alphabetically were omitted from this analysis. The mean $\mathrm{z}$ scores were .60 for List $\mathrm{C} / \mathrm{A}$ and .71 for List $\mathrm{A} / \mathrm{C}$, both significant at the .01 level. The fact that items of the same class tended to be recalled together probably accounts for the fact that the ITR scores were higher for mixed than for unmixed lists.

\section{Retention Tests}

The mean scores on the first trial of the retention test are shown in Table 4 . There were only minor variations in performance on the immediate test. After a l-week interval, recall was consistently higher for concrete than for abstract words. In the case of the critical subsets of the mixed lists, both the main effect of item class and the Item Class by Retention Interval interaction are significant $[\mathrm{Fs}(1,60)=5.07$ and $4.13, \mathrm{p}<.05$ for both $]$. The differences are of the same order of magnitude for the critical subsets of the unmixed lists, but both sources of variance fall short of significance $(F s=3.89$ and 3.10). There are no significant interactions involving type of list (all Fs $<1$ ).

So far, the results show better retention of $\mathrm{C}$ than of A items under both conditions of learning, although the statistical support for this conclusion is firm only in the case of the mixed lists. The picture is, however, complicated by the fact that for the total unmixed lists, the retention differences are rather small, with both the main effect of item class and the Item Class by Retention Interval interaction falling far short of significance $[F s(1,60)=.68$ and 1.42 , respectively $]$. The reason for this discrepancy lies in the pattern of differences between critical and noncritical subsets on the delayed test for unmixed lists. In List A the critical items were retained less well than the noncritical ones (8.94 vs 10.31), whereas the opposite was true in List C $(11.13$ vs 10.18$)$. Note the virtual identity of the scores for the noncritical subsets which contrasts sharply with the rather sizable separation between the critical ones. There are no known systematic reasons for these trends. Words were, of course, balanced across subsets. The mean numbers of correct recalls per item during acquisition were closely comparable for the two subsets in each list: Group C.D-3.67 and 3.73; Group
A-D -4.88 and 4.88. Consequently, these variations in retention must be regarded as chance fluctuations which fortuitously serve to widen the separation between concrete and abstract words when the critical subsets are considered by themselves.

There were only minor changes on the second and third trials of the immediate test; performance on the first trial was close to the terminal level of acquisition and remained stable thereafter. On the delayed test, where there was considerable opportunity for improvement, the changes were more substantial. The changes on the second and third trials, as compared with the first, were averaged for each $\mathrm{S}$. The mean gain scores were as follows: C-D-t.91 (critical subset-+.38); A-D-+1.16 (critical subset-+.84); C/A-D-+.16; A/C-D-+.22. There are no significant differences in the amounts of gain between the two classes of items. In the overall analysis of the critical scores, the difference between the two types of list approaches but does not reach significance $[F(1,60)=3.72, p>.05]$. The Item Class by Type of List interaction is not significant. In general, the amount of gain varied inversely with the level of initial recall. The forced recall procedure in the final phase of the retention test yielded only occasional gains in correct responses.

In view of the operations used to control degree of learning, the evaluation of retention differences in the mixed-list condition was based on comparisons of the critical subsets in Lists $\mathrm{C} / \mathrm{A}$ and $\mathrm{A} / \mathrm{C}$. It is also useful to examine the differences between the critical and the noncritical subsets within the mixed lists. The question of interest here concerns the effects on retention of variations in the level of acquisition of the two subsets. Since criterion was reached faster on $\mathrm{C}$ than on $\mathrm{A}$ items, the discrepancy in the terminal level of performance depended on which class of words was critical. In List $\mathrm{C} / \mathrm{A}$ the mean scores on the criterial trial were 14.59 for $\mathrm{C}$ and 12.47 for $\mathrm{A}$; in List $\mathrm{A} / \mathrm{C}$ the corresponding values were 14.38 and 14.34 . Furthermore, the frequencies of correct responses in learning varied directly with trials to criterion: the mean numbers of correct responses were 54.88 for $\mathrm{C}$ and 48.81 for $\mathrm{A}$ during the acquisition of List $\mathrm{C} / \mathrm{A}$; these measures rose to 83.25 and 74.13 , respectively, in the case of List $\mathrm{A} / \mathrm{C}$.

The retention scores for each subset are presented in Table 5. The values on the immediate test reflect the

Table 4

Mean Scores on First Trial of Retention Test

\begin{tabular}{|c|c|c|c|c|c|c|}
\hline \multirow{3}{*}{$\begin{array}{l}\text { Item } \\
\text { Class } \\
\end{array}$} & \multicolumn{4}{|c|}{ Unmixed Lists } & \multirow{2}{*}{\multicolumn{2}{|c|}{$\frac{\text { Mixed Lists }}{\text { Critical Subset }}$}} \\
\hline & \multicolumn{2}{|c|}{ Total } & \multicolumn{2}{|c|}{ Critical Subset } & & \\
\hline & I & $\mathrm{D}$ & I & $\mathrm{D}$ & 1 & $\mathrm{D}$ \\
\hline $\mathrm{C}$ & 29.38 & 21.31 & 14.88 & 11.13 & 14.56 & 10.31 \\
\hline A & 29.75 & 19.25 & 14.75 & 8.94 & 14.44 & 7.88 \\
\hline
\end{tabular}

Note-MS $S_{E}$ are 16.75 for total unmixed lists, 5.49 for critical subsets of unmixed lists, and 5.18 for critical subsets of mixed lists. 
terminal levels of performance. For a given class of items, the delayed scores are in turn correlated with the immediate ones. Further examination reveals an interesting invariance in the relative amounts retained over the 1-week interval. When the delayed scores are expressed as percentages of the immediate ones, the following values are obtained: critical $\mathrm{C}-71$; noncritical C-70; critical A-55; noncritical A-55. This invariance is found in spite of the large difference between the two lists in the absolute numbers of correct responses during acquisition. There appears to be a limited potential for retrieval of the items from the two subsets, with the difference between them determined by the relative frequencies of correct responses in original learning.

For the measures shown in Table 5, the Item Class by Retention Interval interaction is significant at the .01 level $[F(1,60)=9.66]$. However, the higher-order interaction which includes list (C/A vs $\mathrm{A} / \mathrm{C})$ as a factor is clearly not significant $(\mathrm{F}<1)$. This analy sis confirms the conclusion that the difference between the two subsets in each list was independent of the absolute amount of practice during acquisition.

\section{Organization of Output on Test of Retention}

To determine the extent to which the subjective organization developed during acquisition persisted on the test of retention, ITRs were measured between the last trial of original learning and the first trial of the test of retention. The relative scores, calculated as before, are presented in Table 3. Under all conditions, the ITR values were lower for the delayed than for the immediate test trial, i.e., there was some dissipation over time of the original subjective groupings. This decline is significant at the .02 level for the mixed lists but falls short of significance for the unmixed lists $[F s(1,60)=$ 6.62 and 3.84 , respectively]. There were only minor differences between Lists $\mathrm{C}$ and $\mathrm{A}$, on the one hand, and Lists $\mathrm{C} / \mathrm{A}$ and $\mathrm{A} / \mathrm{C}$, on the other. As was true for the last two learning trials, the ITR scores were higher for mixed than for unmixed lists, but in the present comparison this difference fails to reach significance $[F(1,120)=$ 3.85 , whereas 3.94 is required for the .05 level] .

The frequency of runs was again used to assess clustering by item class in the recall of the mixed lists. The mean $\mathrm{z}$ scores on the immediate test are -1.06 for List $\mathrm{C} / \mathrm{A}$ and -1.21 for List $\mathrm{A} / \mathrm{C}$, both of which are significant at the .01 level. The corresponding values on the delayed test show a sharp drop to -.42 and -.15 , neither of which is significantly different from zero. The decline in the amount of clustering is significant at the .01 level.

\section{DISCUSSION}

In agreement with previous results, free recall learning was found to be faster for concrete than for abstract words. A potentially important qualification has to be added to this conclusion. In the early stages of acquisition, the difference between the two classes of
Table 5

Mean Retention Scores for Critical and Noncritical Subsets in Mixed Lists

\begin{tabular}{crrrrr} 
& \multicolumn{2}{c}{ List C/A } & \multicolumn{2}{c}{ List A/C } \\
\cline { 3 - 5 } Subset & I & D & & I & D \\
\hline C & 14.56 & 10.31 & 14.31 & 10.06 \\
$\mathrm{~A}$ & 12.94 & 7.06 & 14.44 & 7.88 \\
\hline
\end{tabular}

Note-Scores on critical subsets are shown in italics; $M S_{E}=3.21$.

items was greater for mixed than for unmixed lists, reaching significance only under the former condition. The relevant Item Class by List Type interactions were, however, not significant, which is perhaps not too surprising since the differences were consistently in the same direction. Thus, the question of whether the concreteness effect is substantially exaggerated in mixed-list learning cannot be answered definitively on the basis of the present results. ${ }^{1}$ It would be unwise, nevertheless, to ignore the indications that Ss' learning preferences enhance the initial advantage of concrete items when mixed lists are used.

If Ss do, indeed, choose to attend to concrete before abstract items in a mixed list, what might be the reason for their preference? One reasonable supposition is that concrete words are perceived as more distinctive and hence more easily retrievable. Such an interpretation is consistent with the finding of Galbraith and Underwood (1973) that concrete words have lower phenomenal frequency than abstract ones and are also judged to occur in fewer different contexts. These judgments imply a difference in perceived distinctiveness: diversity of connotations should be directly related to phenomenal frequency and contextual variety. The hypothesis that C-I value and item distinctiveness are correlated has, of course, been considered previously, primarily in the context of paired-associate learning (cf. Paivio, 1971, Chap. 8). The present suggestion is not, however, that the positive effects of concreteness on acquisition are attributable to item distinctiveness, but rather that the latter characteristic may form the basis of Ss' learning preferences under mixed-list conditions.

The fact that the measures of early learning yielded small and nonsignificant differences for the unmixed lists calls into question the assumption that the stored representations of concrete words are inherently more memorable than those of abstract words. These results also pose a difficulty for the dual-coding interpretation, at least with respect to its validity for early learning trials. The hypothesis that interitem associations can be activated more readily among concrete than among abstract words remains a reasonable possibility in light of the fact that the terminal criterion was reached faster for concrete than for abstract words under the unmixedas well as the mixed-list arrangement. Such a difference should become increasingly important as learning progresses. It is true that the terminal measures of output consistency were approximately the same for Lists $\mathrm{C}$ and $\mathrm{A}$, but there were more trials on the latter 
than on the former. The fact that for mixed lists there was substantial clustering by item class indicates that C-I value was one of the functional bases of subjective organization. The question remains open of whether imaginal encoding is responsible for whatever advantage concrete words might have with respect to the establishment of higher-order groupings. Alternative explanations warrant consideration. For example, low contex tual variety, which Ss attribute to concrete words, might favor consistent encoding and hence the rapid development of a stable organization.

The measures of retention present an equivocal picture. When the total unmixed lists are considered, the difference in the amount of forgetting is small and far from significant. However, the relative differences are clearly larger for the critical subsets under both conditions of learning. The magnitude of the concreteness effect is comparable for unmixed and mixed lists, although the Item Class by Retention Interval interaction reaches significance only in the latter case. Analysis of the relevant learning data failed to suggest a reason for the divergence of the critical subsets in the unmixed lists. Unsystematic variations probably have to be expected when retention has to be measured for an arbitrary portion of a homogeneous list. For this reason, it may be appropriate to pay special attention to the contrast between the results obtained with total unmixed lists, on the one hand, and with mixed lists, on the other. If that is done, it appears that retention differences are more substantial after mixed- than after unmixed-list learning.

On a delayed retention test for a mixed list, the concrete words may become relatively dominant by virtue of their greater "strength," i.e., because they were recalled more frequently than the abstract ones during original learning. It is known that in free recall the presence of strong items can inhibit the reproduction of relatively weak ones. Thus, the inclusion of repeated items in a list lowers the probability of recall of items presented once (Tulving \& Hastie, 1972); prior study of selected members of an exhaustive category lowers recall for the remaining members (Brown, 1968). It is reasonable to suppose that a similar process comes into play in the delayed recall of a mixed list containing subsets acquired at different rates. The importance of relative strength is underscored by the fact that the difference between the subsets within the mixed lists is independent of the absolute level of acquisition.

The burden of explanation for the observed retention differences cannot be placed entirely on retrieval processes peculiar to mixed lists. On the face of it, there was a concreteness effect for unmixed lists as well, and the statistical analyses fail to show an interaction of retention loss with type of list. No strong conclusions can be drawn from the present results about the processes responsible for the apparent positive relation between word concreteness perse and long-term retention. The measures of output consistency give no indication that the persistence of subjective organization is a function of $\mathrm{C}$-I value. These measures probably have limited validity as indices of organization, and to that extent the evidence is not decisive. It is possible that dual encodings of concrete words are fully developed at the end of acquisition and thereafter provide complementary retrieval routes which are not lost concomitantly. If so, the probability of continued access would be higher for concrete than for abstract words. Nor can a difference in inherent memorability between the representations of the two classes of items be definitely ruled out. However that may be, the results obtained with total unmixed lists suggest that the enhancement of retention tied to the attribute of concreteness as such is not of major proportions.

In general, the present findings call for caution in the evaluation of the effects of $\mathrm{C}$-I value on the acquisit on and retention of free recall lists. A methodological implication worthy of emphasis is that results obtained with mixed lists are not likely to provide a good basis for generalizations about the influence of item attributes on the processes of storage and retrieval.

\section{REFERENCES}

Begg, I. Recall of meaningful phrases. Journal of Verbal Learning \& Verbal Behavior, 1972, 11, 431-439.

Begg, I., \& Robertson, R. Imagery and long-term retention. Journal of Verbal Learning \& Verbal Behavior, 1973, 12, 689-700.

Bousfield, A. K., \& Bousfield, W. A. Measurement of clustering and of sequential constancies in repeated free recall. Psychological Reports, 1966, 19, 935-942.

Brown, J. Reciprocal facilitation and impairment of free recall. Psychonomic Science, 1968, 10, 41-42.

Frankel, F., \& Cole, M. Measures of category clustering in free recall. Psychological Bulletin, 1971, 76, 39-44.

Galbraith, R. C., \& Underwood, B. J. Perceived frequency of concrete and abstract words. Memory \& Cognition, 1973, 1, 56-60.

Paivio, A. Imagery and verbal processes. New York: Holt. Rinehart \& Winston, 1971.

Paivio, A. Imagery and long-term memory. In R. A. Kennedy and A. Wilkes (Eds.), Studies in long-term memory. New York: Wiley, in press.

Paivio, A., \& Csapo, $K$. Concrete-image and verbal memory codes. Journal of Experimental Psychology, 1969, 80, 279-285.

Paivio, A., \& Csapo, K. Picture superiority in free recall: Imagery or dual coding? Cognitive Psychology, 1973, 5, 176-206.

Paivio, A., Yuille, J. C., \& Madigan, S. Concreteness, imagery, and meaningfulness values for 925 nouns. Journal of Experimental Psychology Monograph Supplement, 1968, 76(1, Pt. 2).

Paivio, A., Yuille, J. C., \& Rogers, T. B. Noun imagery and meaningfulness in free and serial recall. Journal of Experimental Psychology, 1969, 79, 509-514.

Postman, L. Transfer, interference and forgetting. In $\mathrm{J}$. W. Kling and L. A. Riggs (Eds.), Experimental psychology. New York: Holt, Rinebart \& Winston, 1971.

Postman, L. Does imagery enhance long-term retention? Bulletin of the Psychonomic Society, 1974, 3, 375-377.

Postman, L., \& Burns, S. Experimental analysis of coding processes. Memory \& Cognition, 1973, 1, 503-507.

Tulving, E., \& Hastie, R. Inhibition effects of intralist repetition in free recall. Journal of Experimental Psychology, 1972, 92, 297-304.

Underwood, B. J. The representativeness of rote verbal learning. In A. W. Melton (Ed.), Categories of human learning. New York: Academ ic Press, 1964

\section{NOTE}

1. Available evidence supports the conclusion that under unmixed-list conditions, the difference between concrete and abstract words is not a robust one. For example, in contrast to the results of Paivio, Yuille, and Rogers (1969), the difference reported by Paivio and Csapo (1969) is quite small, at a slow as well as at a fast rate of presentation. The conditions which are likely to favor a difference remain to be specified.

(Received for publication February 4, 1974; revision received April 15, 1974.) 